\title{
PREVALÊNCIA DE EXAMES DIAGNÓSTICOS DE CÂNCER DE PRÓSTATA EM COMUNIDADE RURAL
}

Rosana Amora Ascari¹, Scheila Pelissari², Márcia Danieli Schmitt ${ }^{3}$, Olvani Martins da Silva ${ }^{1}$, Eliana Buss ${ }^{1}$, Tania Maria Ascari ${ }^{4}$

RESUMO: Estudo descritivo com abordagem quantitativa objetivou identificar a prevalência de exames preventivos de câncer de próstata em homens acima de 40 anos de idade, residentes em uma comunidade no interior de Videira - Santa Catarina. Toda a população da comunidade foi mapeada, totalizando 210 indivíduos e destes, 70 homens que se enquadraram nos critérios de inclusão. Os dados foram coletados por meio de questionário. Constatou-se que $61,8 \%$ dos homens conhecem os exames de prevenção do câncer de próstata; 41,1\% realizam os exames e 58,9\% não realizam, justificando a falta de conhecimento, não julgar necessário e não apresentar sintomas. Foi possível traçar um plano de ações educativas na comunidade acerca do câncer de próstata, sugerindo maior atenção na abordagem à saúde da população masculina. DESCRITORES: Neoplasias da próstata; Diagnóstico precoce; Saúde do homem.

\section{PREVALENCIA DE EXÁMENES DIAGNÓSTICOS DE CÁNCER DE PRÓSTATA EN COMUNIDAD RURAL}

RESUMEN: Estudio descriptivo con abordaje cuantitativo cuya finalidad fue identificar la prevalencia de exámenes preventivos de cáncer de próstata en hombres de más de 40 años de edad, residentes en una comunidad en el interior de Videira - Santa Catarina. Toda la población de la comunidad fue mapeada, totalizando 210 individuos y de estos, 70 hombres que se encuadraran en los criterios de inclusión. Los datos fueron obtenidos por medio de cuestionario. Se constató que $61,8 \%$ de los hombres conocen los exámenes de prevención del cáncer de próstata; $41,1 \%$ realizan los exámenes y 58,9\% no realizan justificando la falta de conocimiento, no juzgar necesario y no presentar síntomas. Fue posible trazar un plan de acciones educativas en la comunidad acerca del cáncer de próstata, sugiriendo más atención en el abordaje a la salud de la población masculina. DESCRIPTORES: Neoplasias de la próstata; Diagnóstico precoz; Salud del hombre.

\section{THE PREVALENCE OF PROSTATE CANCER DIAGNOSTIC TESTS IN A RURAL COMMUNITY}

\begin{abstract}
This descriptive study with a quantitative approach aimed to identify the prevalence of preventive tests for prostate cancer in men aged over 40 years old, resident in the community of Videira in the interior of the State of Santa Catarina. The whole population of the community was mapped, totalling 210 individuals; of these, 70 men met the inclusion criteria. The data were collected using a questionnaire. It was determined that $61.8 \%$ of the men knew about the tests for preventing prostate cancer; $41.1 \%$ had undertaken the tests and $58.9 \%$ had not, stating the cause to be lack of knowledge, not considering it to be necessary, and not presenting symptoms. It was possible to outline a plan of educational actions in the community regarding prostate cancer, suggesting greater attention in the approach to the health of the male population. DESCRIPTORS: Prostate cancers; Early diagnosis; Men's health.
\end{abstract}

\footnotetext{
${ }^{1}$ Enfermeira. Mestre em Saúde Coletiva. Professora da Universidade do Estado de Santa Catarina. Videira-SC-Brasil

${ }^{2}$ Enfermeira. Auditora Interna do Hospital Divino Salvador. Videira-SC-Brasil

${ }^{3}$ Enfermeira. Videira-SC-Brasil

${ }^{4}$ Enfermeira e Psicóloga. Mestre em Enfermagem. Professora da Universidade do Estado de Santa Catarina. Videira-SC-Brasil
} 


\section{INTRODUÇÃO}

O Câncer de Próstata (CP) é frequente em quase todas as regiões do país e, no decorrer dos anos, observa-se tendência de aumento de novos $\operatorname{casos}^{(1)}$. Considerando ser uma neoplasia que apresenta evolução lenta, a mortalidade pode ser evitada com diagnóstico e tratamento precoce ${ }^{(2)}$. Na última estimativa mundial, realizada em 2012, o CP foi referido como o segundo câncer mais frequente entre os homens, sendo que $75 \%$ dos casos diagnosticados ocorrem em países desenvolvidos ${ }^{(3)}$. No Brasil, são vários os fatores que justificam o aumento das taxas de incidência no decorrer dos anos, como o aumento da expectativa de vida, melhoria e evolução dos métodos de diagnóstico e qualidade dos sistemas de informação $0^{(3)}$.

Todo homem, a partir de 45 anos de idade deve realizar consulta preventiva; aqueles que possuem antecedentes familiares de câncer de próstata deverão iniciar a prevenção a partir dos 40 anos de idade. A avaliação prostática preventiva possibilita a detecção de tumores prostáticos localizados, em fase inicial de desenvolvimento, oferecendo chances reais de cura aos pacientes $^{(4)}$.

A idade é o único fator de risco bem estabelecido para o desenvolvimento do CP, bem como a raça/etnia, o histórico familiar, dietas ricas em gordura animal, carne vermelha, embutidos e cálcio, a obesidade e a síndrome metabólica, caracterizada pela resistência do hormônio insulina ${ }^{(3)}$.

O CP é mais incidente na região Sudeste $(78 / 100$ mil), e Nordeste (43/100 mil) do Brasil. Não considerando os tumores da pele não melanoma, é também o mais frequente nas regiões Centro-Oeste (75/100 mil), Sul (68/100 mil) e Norte (30/100mil). No Estado de Santa Catarina, estima-se 1670 novos casos por $100 \mathrm{mil}$ habitantes no ano de 2012, e na capital Florianópolis, 70 casos novos por 100 mil habitantes, conforme as taxas de incidência e localização primária ${ }^{(3)}$.

Com o aumento das estatísticas no Brasil de mortalidade por neoplasias, a Política Nacional de Prevenção e Controle do Câncer foi elaborada pelo Ministério da Saúde com o objetivo de reduzir a incidência e mortalidade por câncer, visando ações de conscientização da população quanto aos fatores de risco, promovendo a detecção precoce dos cânceres, como também proporcionar um tratamento de qualidade em todo o país ${ }^{(5)}$.

Neste sentido, a Lei n. 10.289, de 20 de setembro de 2001, institui o Programa Nacional de Controle do Câncer de Próstata, que inclui atividades como cam- panhas esclarecendo o que é câncer de próstata e suas formas de prevenção e em parceria com as Secretarias Municipais e Estaduais de Saúde, disponibilizando aos homens acima de 40 anos exames de prevenção ao $\mathrm{CP}^{(6)}$.

A elaboração da Política Nacional de Atenção Integral à Saúde do Homem (PNASH) tem como objetivo promover a melhoria das condições de saúde dessa população em todo o país, visando reduzir a morbidade e mortalidade, enfrentando os fatores de risco e facilitando o acesso da população masculina às ações e serviços de assistência integral a saúde ${ }^{(2)}$. Também busca orientar as ações e serviços de saúde voltada à população masculina, para que haja mudança dos atuais paradigmas, integrando a execução da presente política às demais políticas, programas, estratégias e ações do Ministério da Saúde ${ }^{(2)}$.

Aumentar a expectativa de vida, reduzir a morbimortalidade por causas evitáveis, promover e incentivar a prática de cuidados com a saúde masculina e implementar as metas estabelecidas pela PNASH consistem em desafios para os gestores das políticas de saúde $\operatorname{propostos}^{(7)}$.

A carência de estudos que abordem, especificidades, a saúde do homem, especialmente que focalizem o CP, já foi sinalizada. Há necessidade da realização de novas pesquisas para avaliar o conhecimento que os homens têm sobre os exames de rastreamento desse câncer e em que circunstâncias esses exames são realizados ${ }^{(8)}$.

Considerando a importância do tema em questão, faz-se a seguinte pergunta: "Qual a prevalência de homens acima de 40 anos, residentes numa comunidade rural do Município de Videira - Santa Catarina, que realizam periodicamente os exames diagnósticos do câncer de próstata?".

Os objetivos do estudo foram identificar quantos homens acima de quarenta anos de idade realizam, periodicamente, os exames diagnósticos do câncer de próstata, quantos não realizam e quais os fatores que impedem a procura dos mesmos; e realizar intervenção educativa aos participantes da pesquisa por meio de oficina.

\section{MÉTODO}

Pesquisa-intervenção descritiva com abordagem quantitativa, desenvolvida em uma comunidade rural no interior de Videira - Santa Catarina no período de fevereiro a agosto de 2008. No primeiro momento, foi realizado um mapeamento da localidade identifican- 
do a população residente no local. O público-alvo foi representado por homens acima de 40 anos de idade, residente na área de estudo. Foram excluídos os institucionalizados ou impossibilitados de darem respostas coerentes com o objeto de estudo.

Numa população de 210 indivíduos do sexo masculino, 70 homens que se enquadraram no critério de inclusão, destes, 68 concordaram em participar do estudo assinando o Termo de Consentimento Livre e Esclarecido. Os dados coletados foram registrados em fichas individuais contendo dados sobre a idade, conhecimento acerca dos exames diagnósticos de $\mathrm{CP}$, fatores que impedem ou dificultam sua realização, a periodicidade quanto a realização, presença ou não de sinais e sintomas característicos de alterações prostáticas, além da exposição a fatores de risco para o desenvolvimento de neoplasias.

Os participantes tiveram acesso aos resultados da pesquisa através de uma oficina de conscientização na própria comunidade, na qual foram abordados sobre os exames diagnósticos do CP e a importância da realização dos mesmos para a saúde do homem.

A pesquisa foi desenvolvida de acordo com os aspectos éticos envolvendo seres humanos e aprovada conforme parecer consubstanciado CEPSH/UNOESC/ HUST n. 148/2007.

Quanto a análise estatística, os dados foram digitados em planilha do Programa Microsoft Excel receberam tratamento estatístico e as variáveis categóricas foram representadas através de números absolutos.

\section{RESULTADOS}

Após a tabulação dos dados constatou-se que $44,2 \%(\mathrm{n}=30,05)$ dos participantes da pesquisa estavam entre 40 a 49 anos de idade; $33,8 \%(n=22,9)$ entre 50 a 59 anos; $14,8 \%(n=10,06)$ entre 60 a 69 anos e $7,2 \%$ $(n=4,8)$ com faixa etária dos 70 aos 84 anos de idade.

Quando questionados sobre quais são os exames indicados para diagnóstico precoce de CP, 61,8\% afirmaram conhecer. Acerca do conhecimento específico sobre o exame sanguíneo de dosagem Antígeno Prostático Específico (PSA), 57,3\% $(n=38.9)$ responderam conhecer. Quando abordado sobre o exame retal digital (ERD), $75 \%$ dos participantes alegaram ter conhecimento.

A pesquisa identificou que $58,9 \%$ não realizaram nenhuma vez os exames e $41,1 \%$ algum exame para diagnóstico de CP. De 28 homens que realizaram algum exame para diagnóstico precoce do $\mathrm{CP}, 12$ foram para o PSA e o ERD; 10 somente PSA e seis apenas o ERD.
Segundo dados informados no instrumento de coleta, os homens que nunca realizaram exames para diagnóstico precoce de $\mathrm{CP}$ justificaram ser por acomodação, falta de incentivo, falta de conhecimento, por não achar necessário, falta de interesse, pouca idade e por falta de divulgação dos exames, além de não apresentar nenhum sintoma. Ao questionar aqueles que não realizaram os exames diagnósticos, se ainda pensam em realizar, $94,2 \%(n=37)$ afirmaram que sim.

Esses participantes reconhecem que os exames de PSA e ERD deveriam ser realizados para o diagnóstico precoce, mesmo não os fazendo. De acordo com tempo do último exame, $1,4 \%$ realizaram seu exame de diagnóstico no ano de $1986 ; 1,4 \%$ no ano de 1997 ; $1,4 \%$ em 2004; $1,4 \%$ em 2005; $9 \%$ no ano de 2006 ; $17,7 \%$ em 2007 e 10,3\% em 2008. Nunca realizaram os exames $57,4 \%$. A pesquisa aponta que 50 pessoas não realizam anualmente consulta com urologista $\mathrm{e}$ destacam a falta de incentivo, por acomodação, por não ter motivo e por não apresentar sintoma.

\section{DISCUSSÃO}

Em todo o mundo $62 \%$ dos casos identificados de CP acometem homens acima de 65 anos e, até no ano de 2015, com o aumento da estimativa de vida, é esperado um acréscimo de $60 \%$ dos casos novos de $\mathrm{CP}^{(3)}$. Outro estudo aponta a incidência de CP aumenta, significativamente, depois dos 50 anos de idade, e mais de $70 \%$ dos casos acontecem em homens acima de 65 $\operatorname{anos}^{(9)}$.

Dentre os exames preventivos do CP, o teste PSA e o ERD são os mais indicados para diagnóstico precoce. Esses métodos diagnósticos como o toque retal e o PSA sérico são indicados anualmente, pois além do baixo custo, possui especificidade e sensibilidade ${ }^{(10)}$.

O PSA é uma glicoproteína que se origina na próstata, quando seu nível se eleva na corrente sanguínea é considerado como um marcador biológico para algumas doenças prostáticas, inclusive o câncer. $\mathrm{O}$ toque retal avalia o tamanho, a forma e a consistência da próstata, com o objetivo de constatar presença de nódulos ${ }^{(7)}$. Se por um lado recomendam-se tais práticas como medidas de detecção precoce do $\mathrm{CP}$, por outro, se evidência que a dosagem de marcadores como o PSA quando utilizada como forma de rastreamento de pacientes, não está ligado diretamente com a redução da mortalidade e, por este motivo, vários homens estão se submetendo a cirurgias desnecessárias ${ }^{(11)}$. O teste PSA 
também apresenta algumas limitações, em especial a baixa especificidade. Hiperplasia prostática benigna, infecção e inflamação crônica podem elevar o nível de PSA, levando ao resultado como um falso positivo ${ }^{(1)}$.

Muitos homens possuem receio de realizar o exame de toque retal, o que gera preocupação, pois a falta de diagnóstico, ou o diagnóstico tardio, tem ocasionado à morte. E homens acima de 50 anos de idade, a realização do PSA é essencial para o rastreamento precoce do $\mathrm{CP}$, observando também que o toque retal concomitante ao PSA reafirma a detecção do câncer ${ }^{(8)}$.

Os participantes de um estudo tiveram dúvida quanto ao principal exame para prevenção do $\mathrm{CP}$, onde o toque retal foi citado pela maioria, seguido dos que consideraram necessários os exames de toque retal e o PSA, concomitantemente, e alguns participantes acreditavam ser o teste suficiente ${ }^{(12)}$. No presente estudo constatou-se a que a procura por estes exames não foi expressiva, considerando que menos da metade dos participantes já realizaram exames para detecção precoce do CP.

Estudo aponta como causa da não realização do exame de toque retal, o fato do médico nunca ter solicitado, pela amostra se considerar saudável, descuido/ esquecimento, falta de tempo, por confiança no exame do PSA e por medo. Em estudo realizado com 88 gaúchos tradicionalistas, $83 \%$ realizaram ao menos uma vez, um dos exames preventivos do $\mathrm{CP}^{(12)}$. No Estado de São Paulo ${ }^{(7)}$ foram analisados os dados de 992 homens com 50 anos ou mais de idade, e verificou-se que quase metade da população estudada nunca havia realizado o exame preventivo ${ }^{(12)}$.

Percebe-se que não há esclarecimento suficiente sobre os exames para diagnóstico precoce junto aos participantes, uma vez que se confundem quando questionados a respeito do assunto. Não basta dispor de uma Política Nacional de Prevenção de Controle do Câncer de Próstata, visto que por si só ela não está sendo eficaz.

Um dos fatores responsáveis pelo diagnóstico tardio da doença se deve à falta de informação da população, além da inexistência de procedimentos específicos e sensíveis que possam detectar o tumor em fase microscópica e à dificuldade de implantação de rotinas abrangentes, programadas nos serviços de saúde, que favoreçam a deteç̧ão precoce ${ }^{(4)}$.

Percebe-se que não é rotina a realização de consulta para fins de promoção da saúde, fazendo com que a procura por profissionais da área só ocorra quando realmente há manifestação de sinais e sintomas anor- mais. Os resultados evidenciam a não implementação do Programa Nacional de Controle do Câncer de Próstata, que visa o esclarecimento sobre o que é CP, como também a disponibilização de exames para a sua detecção precoce.

Destaca-se a importância de campanhas educativas, promovendo ações, também com os profissionais de saúde, no intuito de fomentar o conhecimento através de materiais ilustrativos e educativos que facilitem a compreensão e conscientização do público alvo, como forma de promoção da saúde ${ }^{(4)}$. O maior desafio no CP é promover o diagnóstico precoce, pois quando o câncer está restrito apenas à próstata e é curável. Quanto mais precocemente for diagnosticado e tratado, maiores as possibilidades de cura, além do tratamento ser menos agressivo e de menor custo quando comparado ao tratado em estágios mais avançados ou com metástase ${ }^{(5)}$.

A promoção da saúde da população, como a ação da atenção primária à saúde auxilia no processo do diagnóstico precoce. Estar atentos e verificar se os usuários se encontram na idade de iniciar os exames de deteç̧ão precoce de câncer e outras doenças associadas, como também incentivar e sensibilizá-los quanto à importância dos homens aprenderem acerca da manutenção da saúde como um todo, são papéis dos profissionais de saúde ${ }^{(4)}$.

Com incentivos e divulgação de informação talvez a resposta acerca da realização dos exames PSA e ERD para diagnóstico precoce do CP pudesse ser unânime.

\section{CONCLUSÃo}

Esse estudo evidenciou que apenas $41 \%$ dos participantes realizaram em algum momento os exames diagnósticos para $\mathrm{CP}$, sendo que os homens que não realizam justificam tal prática por falta de conhecimento, por não julgarem necessários ou não apresentarem nenhum sintoma. Isso posto, percebe-se uma lacuna na implementação das políticas públicas de saúde que visam prevenir tais agravos à saúde do homem.

Em relação aos meios de diagnóstico precoce do $\mathrm{CP}$, pode-se afirmar que a procura pelos exames de ERD e PSA não são tão expressivo, sendo que mais da metade dos participantes conhece melhor o exame de toque do que o teste sanguíneo, mas não realizaram nenhum destes, apesar de haver evidência científica de que sua realização auxilia no diagnóstico precoce.

Apesar do Programa Nacional de Controle do Câncer de Próstata ter sido instituído em 2001, pouco se sabe sobre a real condição da saúde dos homens brasileiros, especificamente sobre seus conhecimentos 
de prevenção e a realização de exames para diagnóstico precoce do CP, o que abre perspectivas de novos estudos para aprofundamento do tema.

\section{REFERÊNCIAS}

1. Ministério da Saúde (BR). Câncer no Brasil, dados dos registros de base populacional.v.4. Riode Janeiro[Internet] 2010 [acesso em 11 fev 2013]. Disponível: http://www. inca.gov.br/cancernobrasil/2010/docs/registro\%20 de $\% 20$ base $\% 20$ populacional_completo.pdf.

2. Ministério da Saúde (BR). Secretaria de atenção à saúde. Departamento de ações programáticas estratégicas. Política Nacional de Atenção Integral à Saúde do Homem (princípios e diretrizes). Brasília [Internet] 2008 [acesso em 13 fev 2013]. Disponível: http://dtr2001.saude. gov.br/sas/PORTARIAS/Port2008/PT-09-CONS.pdf

3. Instituto Nacional do Câncer - INCA. Estimativa 2012. Incidência de câncer no Brasil. Rio de janeiro [Internet] 2011 [acesso em 11 fev 2013]p.118. Disponível: http://www. inca.gov.br/estimativa/2012/estimativa20122111.pdf

4. Lima ACF, Silva KVM, Caetano JA, Lima MA, Andrade LM. Conhecimento dos trabalhadores de uma universidade privada sobre a prevenção do câncer de próstata. Cogitare enferm. [Internet] 2007;12(4) [acesso em 28 mar 2013]. Disponível: http://ojs.c3sl.ufpr.br/ojs2/ index.php/cogitare/issue/view/671.

5. Instituto Nacional de Câncer. Programa Nacional de controle do Câncer da Próstata documento de consenso. Rio de Janeiro: INCA [Internet] 2002 [acesso em 11 fev 2013]. Disponível: http://bvsms.saude.gov.br/bvs/ publicacoes/cancer_da_prostata.pdf

6. Brasil. Lei $\mathrm{n}^{\circ} 10.289$, de 20 de setembro de 2001. Institui o Programa Nacional de Controle do Câncer de Próstata. [Internet] 2013 [acesso em 11 fev 2013]. Disponível: http://www6.senado.gov.br/legislacao/ ListaPublicacoes.action?id $=233592$

7. Amorim VMSL, Barros MBA, César CLG, Goldbaum M, Carandina L, Alves MCGP. Fatores associados à realização dos exames de rastreamento para o câncer de próstata: um estudo de base populacional. Cad. Saúde Pública. [Internet] 2011;27(2) [acesso em 12 fev 2013]. Disponível: http://dx.doi.org/10.1590/S0102311X2011000200016

8. Barouki MPE. Rastreamento do câncer de próstata em homens acima de 50 anos através do exame diagnóstico de PSA. Rev. Gestão \& Saúde. [Internet] 2012;3(2) [acesso em 12 mar 2013]. Disponível: http://www.gestaoesaude. unb.br/index.php/gestaoesaude/ article/view/142.

9. Thurston A. Câncer de Próstata. Rev. Medical UPDATE. 2003;1(3):21-3.

10. Miranda PSC, Côrtes MCJW, Martins ME, Chaves PC, Santarosa RC. Práticas de Diagnóstico de Câncer de Próstata entre Professores da Faculdade de Medicina UFMG. Rev. Assoc. Med. Bras. [Internet] 2004;50(3) [acesso em 22 out 2007] Disponível: http://dx.doi. org/10.1590/S0104-42302004000300033

11. Medeiros AP, Menezes MFB, Napoleão AA. Fatores de risco e medidas de prevenção do câncer de próstata: subsídios para a enfermagem. Rev. bras. enferm. [Internet] 2011;64(2) [acesso em $20 \mathrm{fev}$ 2013]. Disponível: http://dx.doi.org/10.1590/S003471672011000200027

12. Souza LM, Silva MP, Pinheiro IS. Um toque na masculinidade: a prevenção do câncer de próstata em gaúchos tradicionalistas. Rev. Gaúcha Enferm. [Internet] 2011;2(1) [acesso em 20 fev 2013]. Disponível: http://dx.doi.org/10.1590/S1983-14472011000100020 